\title{
Adaptive sliding mode control of a novel cable driven robot model
}

\author{
F. Inel ${ }^{1}$ and S. Babesse ${ }^{2}$ \\ ${ }^{1}$ Department of Mechanical Engineering, Laboratory of Automatic, \\ University of Skikda, 21000 Skikda Algeria, \\ Phone: +213671800809 \\ *Email: inelfouad@yahoo.fr \\ ${ }^{2}$ Department of Electrical Engineering, University of Setif1, \\ 19000 Setif, Algeria
}

\begin{abstract}
In this paper, we propose an adaptive sliding mode control strategy for a 3D cable-driven parallel robot. The proposed control technique is widely used for dealing with nonlinear systems uncertainties and for improving the robot performance in terms of tracking a desired path. The main contribution of this work is firstly: the graphical user interface (GUI) witch presents a point-to-point command, thus by the visualization of the end-effector position. Secondly, the sliding mode control is modeling for applied to the dynamic model for different trajectories in order to test the accurate tracking of the robot to a desired path. The effectiveness of the proposed control strategy is demonstrated through different simulation results.
\end{abstract}

Keywords: Cable driven robot; modeling; sliding mode; GUI; position control; robustness.

\section{INTRODUCTION}

Cable driven parallel manipulators are a special class of parallel mechanisms, whose trusts consist of cables having adjustable length to control the end-effector's position and orientation [1-2]. This last is a parallel mechanism in closed chains with $\mathrm{N}$ degrees of freedom for the end effector (figure 1). Cable robots are relatively simple in form, with multiple cables attached to a mobile platform or end-effector. The end-effector is manipulated by motors that can extend or retract the cables [3]. The cables arrangement results in closed chains that can be seen as a parallel mechanism. Considering that cables can only pull, usually a cable driven robot has n-1 degrees of freedom for the end effector with $\mathrm{n}$ being the number of cables, since one cable is usually needed to keep the others in tension. Cable-driven robots are a type of parallel manipulators where the end-effector is supported in parallel by $n$ cables with $n$ tensioning actuators [4]. Cable driven robots have few moving parts with reduced mass and inertia. Accordingly, they are most suitable for tasks requiring high performance such as speed and accuracy, and large workspace, [5-6]. A well-known application of a cable driven system is the Skycam, which can operate a camera in a whole stadium area,[7] Among other applications of cable-driven robots, it is worth to mention haptic interfaces [8] and systems for lifting loads [9]. Some other examples at LARM in 
Cassino are also reported for rehabilitation applications such as in [10] and as a passive tracking systems such as reported in [11]. One of the key aspects for cable driven robots is the need of a proper control strategy to achieve proper motions without breaking the cables. The sliding mode method has been designed to improve the robustness of robotic system control, as reported for example in [12].

In particular, an adaptive sliding mode controller can adjust the control torque based on real-time position tracking error in the set-point control of the end-effector. The paper is organized as follows: the first section present the structure and the mathematical model of our robot.in the second section presents the graphical user interface (GUI) witch presents a point-to-point command and in the last section presents a numerical results simulation.

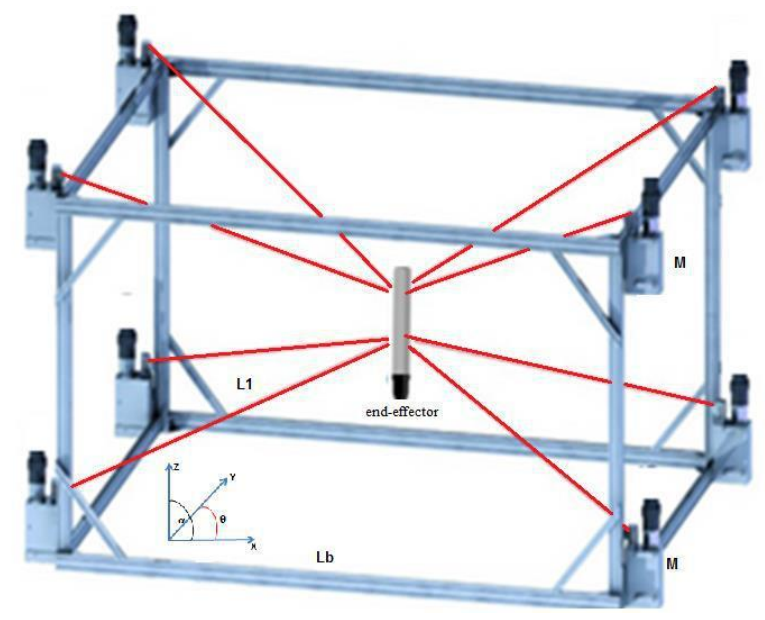

Figure 1. The home-made prototype of spatial cable-based robot with 8 cables.

\section{SYSTEM STRUCTURE AND MODELING}

This section proposes a model of a 3D cables parallel robot that has been designed and built. Figure 2 shows the structure of robot with eight cables [13].

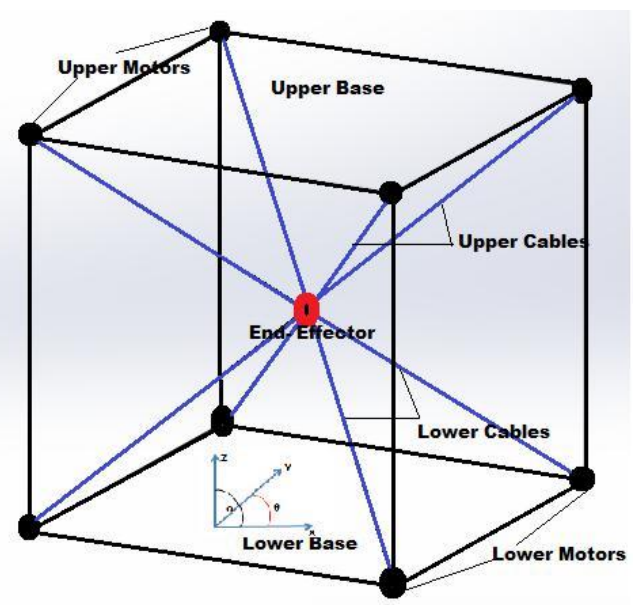

Figure 2. The structure of 3D Cable-Based Robot with 8 cables. 
The elements involved in the scheme of figure 2 are:

$\theta \mathrm{i}$ : the rotational angles of the cables with respect to the $\mathrm{X}$ axis.

$\alpha \mathrm{i}$ : the rotational angles of the cables between the plane $(\mathrm{X}, \mathrm{Y})$ and the axis $\mathrm{Z}$.

In order to analyze the input-output behavior of the cable-based robot under consideration, we exploit the study developed and presented in [14], and [15] where the inverse geometric, the direct geometric, the inverse kinematic and the direct kinematic models are derived as well as the study of the static forces and the dynamic model. Hereafter, we present the dynamic model, which describes the equation of motion of the end-effector. It combines the end-effector and the motor characteristics. It can be represented by a system of two coupled nonlinear differential equations:

$$
M(X) \ddot{X}+N(X, \dot{X})=S(X) \tau
$$

Where:

$$
\tau=\left[\begin{array}{l}
\tau_{1} \\
\tau_{2} \\
\tau_{3} \\
\cdot \\
\cdot \\
\tau_{8}
\end{array}\right]
$$

Is the vector of the torques applied on the cables.and

$$
\begin{gathered}
M=r^{*} m+S(X) J \frac{\delta \beta}{\delta X} \\
N(X, \dot{X})=S(X)\left(J \frac{d}{d t} \frac{\delta \beta}{\delta X}+C \frac{\delta \beta}{\delta X} \dot{X}\right)
\end{gathered}
$$

The relationship between the applied forces acting on the end-effector and the cable tensions $t_{i}$ can be expressed as follows:

$$
F_{R}=S \tau
$$

Where: $F_{R}$ is the resultant force of all tensions applied to the cables and $S$ is the jacobian matrix[16].

$$
\begin{aligned}
& F_{R}=\left(F_{R X} F_{R Y} F_{R Z}\right)^{T}
\end{aligned}
$$

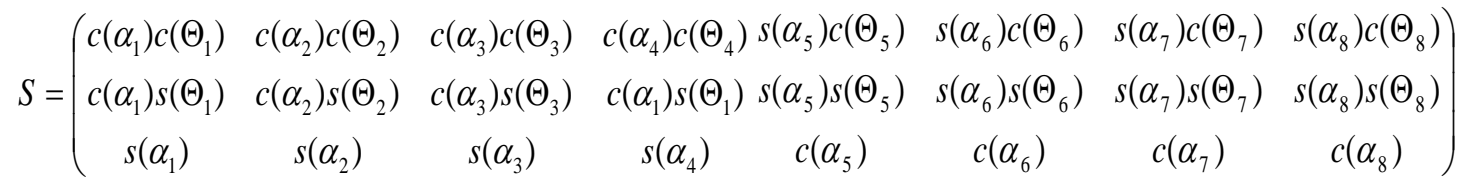


with $c(\theta)$ and $s(\theta)$ represent $\cos (\theta)$ and $\sin (\theta)$ respectively.

To present the dynamical model in the state space form, we introduce the state variables:

$$
\begin{gathered}
\dot{x}_{12 d}(t)=x_{22 d}(t) \\
M_{11} \dot{x}_{22}(t)+M_{12} \dot{x}_{42}(t)+M_{13} \dot{x}_{62}(t)=u_{1}(t)-N_{11} x_{22}(t)+N_{12} x_{42}(t)+N_{13} x_{62}(t) \\
\dot{x}_{32 d}(t)=x_{42 d}(t) \\
M_{21} \dot{x}_{22}(t)+M_{22} \dot{x}_{42}(t)+M_{23} \dot{x}_{62}(t)=u_{2}(t)-N_{21} x_{22}(t)+N_{22} x_{42}(t)+N_{23} x_{62}(t) \\
\dot{x}_{52 d}(t)=x_{62 d}(t) \\
M_{31} \dot{x}_{22}(t)+M_{32} \dot{x}_{42}(t)+M_{33} \dot{x}_{62}(t)=u_{3}(t)-N_{31} x_{22}(t)+N_{32} x_{42}(t)+N_{33} x_{62}(t)
\end{gathered}
$$

where

$$
J \ddot{\beta}+C \dot{\beta}=\tau-r t
$$

We consider identical rays (ri) of the pulley:

$\mathrm{ri}=\mathrm{r}(\mathrm{i}=1.2 \ldots 8)$.

$\beta:$ is the rotation angle of the pulley (like in Fig.3).

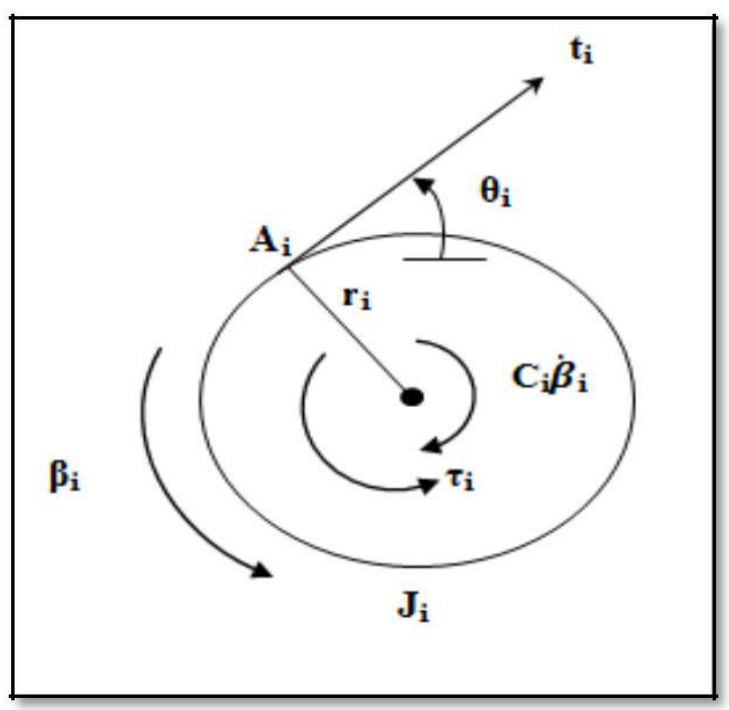

Fig. 3.The structure diagram of the pulley /shaft.

From equation 7:

$$
t=\frac{1}{r}(\tau-J \ddot{\beta}-C \dot{\beta})
$$




$$
\beta=\left(\begin{array}{c}
\beta_{1}(X) \\
\beta_{2}(X) \\
\cdot \\
\cdot \\
\cdot \\
\beta_{8}(X)
\end{array}\right)=\frac{1}{r}\left(\begin{array}{c}
L_{10}-L_{1} \\
L_{20}-L_{2} \\
\cdot \\
\cdot \\
\cdot \\
L_{80}-L_{8}
\end{array}\right)
$$

Where:

$L i=\sqrt{(x-A i x)^{2}+(y-A i y)^{2}+(z-A i z)^{2}} ;$ : the lengths of the cables that connect the base to the mobile platform.

Substituting (8) and (9) we obtain:

$$
t=\frac{1}{r}\left(\tau-J\left(\frac{d}{d t}\left(\frac{\delta \beta}{\delta X}\right) X+\frac{\delta \ddot{\beta}}{\delta X} \ddot{X}\right)-C \frac{\delta \beta}{\delta X} \dot{X}\right)
$$

$\mathrm{t}: \mathrm{t}(\mathrm{t} 1, \mathrm{t} 2, \ldots \mathrm{ti})^{\mathrm{T}}$ : is the vector of tension cables.

Ci: The viscous damping coefficients of each motor shaft.

Ji: The inertia of the rotor and the pulley of each motor.

The state space representation can be derived in the general form.

$$
X(t)=F(X, t)+g(X, t) * U(t)
$$

where :

$$
U(t)=\left[\begin{array}{c}
0 \\
U_{1}(t) \\
0 \\
U_{2}(t) \\
0 \\
U_{3}(t)
\end{array}\right]
$$

$\mathrm{X}(\mathrm{t})$ represents the state space vector $\mathrm{F}(\mathrm{X}, \mathrm{t}), \mathrm{g}(\mathrm{X}, \mathrm{t})$ are nonlinear functions $\mathrm{U}(\mathrm{t})$ represents the command vector. The resulting tension at the end effector leads it to move towards a corresponding position on its workspace. However, to working properly, there is an additional constraint that should be fulfilled concerning the dynamical equilibrium of the end-effector. This means that, at any instant, all the cables should be maintained under minimal and positive tensions to avoid the collapsing of any cable [17]. 


\section{GRAPHICAL USER INTERFACE}

In this section, we present a graphical user interface which is composed of two parts: a 3D schematic view of the proposed robot and the input interface for the position of end-effector as well as modification of the parameters (xref, yref and zref) fig.4.

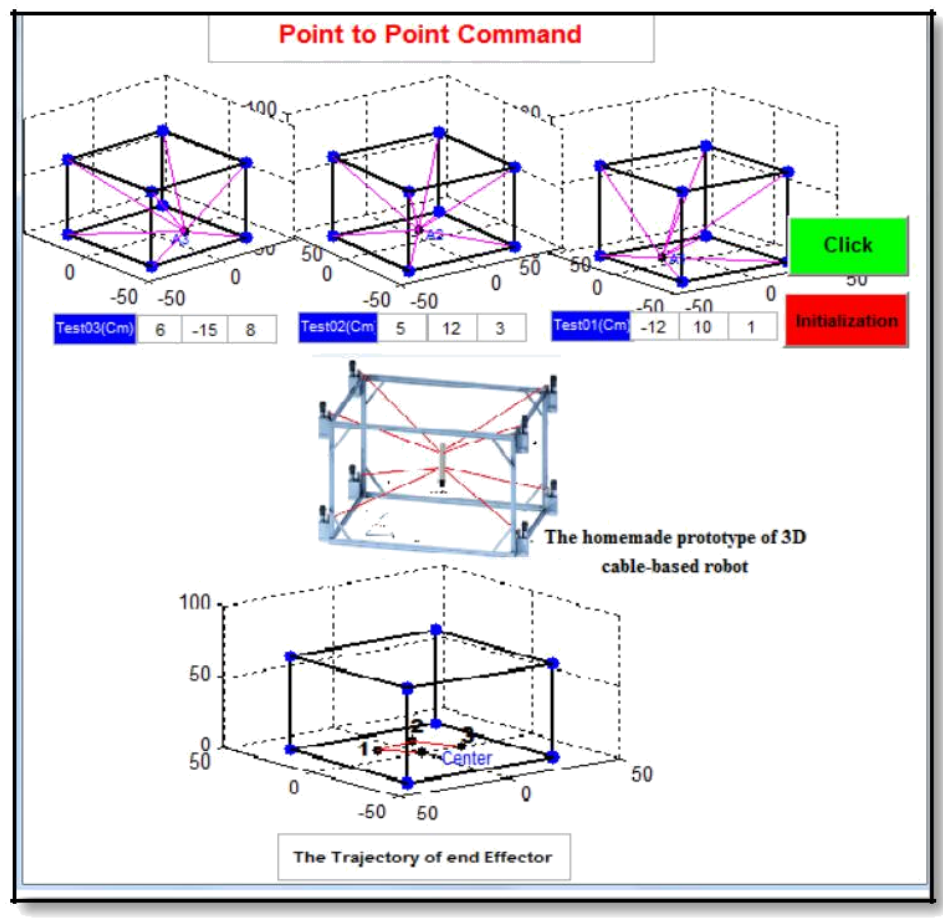

Figure 4. The proposed graphical user interface.

\section{CONTROL LAW STRATEGY}

The robust sliding mode control is to bring the state trajectory to the sliding surface and to switch by means of a switching logic appropriate around it to the balance point. Nevertheless, the SM controller requires a switching law of control, which has the drawback to generate chattering over the controlled system [18]. The main advantage of sliding mode control is that the system is insensitive to extraneous disturbance and internal parameter variations while the trajectories are on the switching surface [19].

To define a sliding mode controller we need to determine an appropriate sliding surface along $\mathrm{x}, \mathrm{y}$ and $\mathrm{z}$. The sliding surface of a common sliding mode controller for a system is generally defined as:

The sliding surface along $\mathrm{x}, \mathrm{y}$ and $\mathrm{z}$ can be expressed as:

$$
\begin{array}{rl}
s_{2 d x}=C_{12 d x} & *\left(x_{12 d}(t)-x_{r e f}\right)+C_{22 d x} * x_{22 d}(t) \\
s_{2 d y} & =C_{12 d y} *\left(x_{22 d}(t)-y_{r e f}\right)+C_{22 d y} * x_{42 d}(t)
\end{array}
$$




$$
s_{2 d z}=C_{12 d z} *\left(x_{32 d}(t)-z_{\text {ref }}\right)+C_{22 d z} * x_{62 d}(t)
$$

$C_{12 d x}, C_{22 d x}, C_{12 d y}$ et $C_{22 d y}, C_{12 d z}$ et $C_{22 d z}$ : are parameters determined by simulation.

Xref, Y ref and Zref are the parameters of desired trajectory planning for the end-effector.

To determine the order of law we worked with new synthesis method that is the approach to the final law [20].

$$
\begin{aligned}
& \dot{S}_{2 d x}=-K_{2 d x} S_{2 d x}-Q_{2 d x} \operatorname{sigh}\left(S_{2 d x}\right) \\
& \dot{S}_{2 d y}=-K_{2 d y} S_{2 d y}-Q_{2 d y} \operatorname{sign}\left(S_{2 d y}\right) \\
& \dot{S}_{2 d z}=-K_{2 d z} S_{2 d z}-Q_{2 d z} \operatorname{sign}\left(S_{2 d z}\right)
\end{aligned}
$$

Where

$K 2 d x, 2 d x, K 2 d y, 2 d y$ : are parameters determined by simulation.

Sign: is the sign of surface (equal to +1 or -1 ).

In our system, we have a non-linear dynamic equation (eq.11), for this purpose, we use a Runge Kutta method because it's suitable for solving non-linear partial differential equations.

The equations of $(U x, U y, U z)$ along $x, y$ and $z$ (combination of equation (6),(11),(13) and $(16) ;(6),(11),(14)$ and (17) and (6), (11), (15) and (18) successively) represent the command vector of the cable-based robot for applying adequate electrical voltages to the motors in order to generate tensions on the cables.

The algorithm of sliding mode in closed-loop case is shown in Fig.5. 


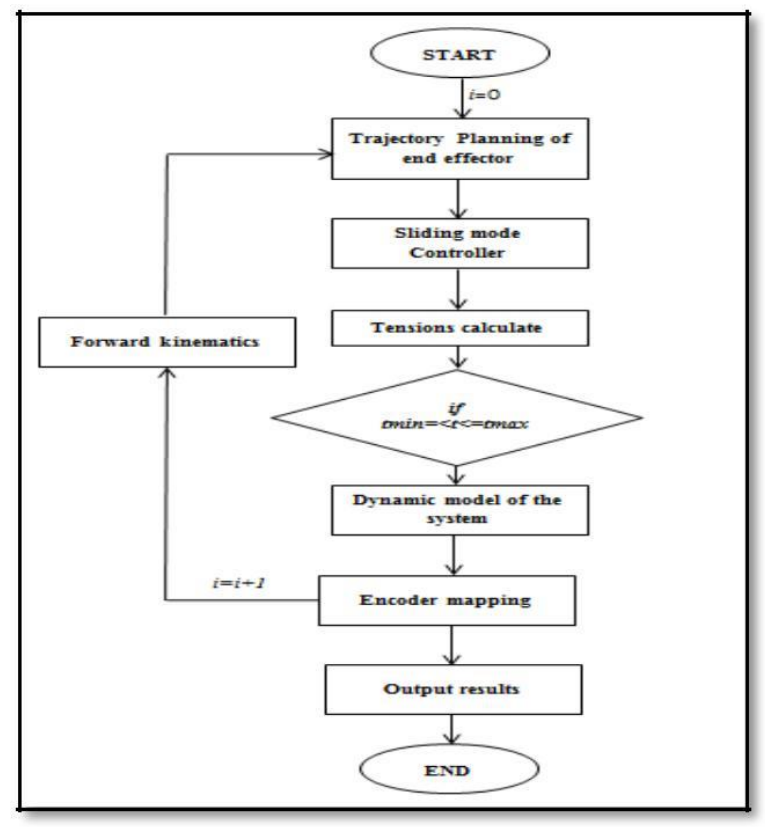

Figure 5. Flow-chart of the sliding mode control algorithm.

\section{SIMULATIONS RESULTS}

In this part, we present the simulation results of 3D cables-based robot with eight cables, but in our system, we do not have direct access to Cartesian position of the end-effector. Instead, we get the direct measurements of the rotation angles $\beta i$ of the pulleys. Then these values are converted into the cable lengths $\mathrm{Li}$, these lengths are then used as inputs to the forward kinematics to obtain the Cartesian position X [20].

The parameters values that provide an acceptable compromise performance have been selected by trial/error for our SMC are:

$C 12 d x=55000 ; C 22 d x=150 ; K 2 d x=150 ; Q 2 d x=100 ; C 12 d y=55000 ; C 22 d y=150$;

$K 2 d y=150 ; Q 2 d y=100 ; C 12 d z=55000 ; C 22 d z=150 ; K 2 d z=150 ; Q 2 d z=100$.

And the parameters for the dynamic equation (1) for our 8 cables robot are mentioned in the following table:

Table 1. Initial values of the robot parameters.

\begin{tabular}{ccc}
\hline Variables & Initial Value & Unit \\
\hline $\mathrm{m}$ & 0.01 & $\mathrm{Kg}$ \\
$\mathrm{C}$ & 0.01 & $\mathrm{Nms}$ \\
$\mathrm{J}$ & 0.0008 & $\mathrm{kgm}^{2}$ \\
$\mathrm{r}_{\mathrm{i}(1,2, \ldots, 8)}$ & 1.5 & $\mathrm{~cm}$ \\
$\mathrm{~L}_{(1,2, . ., 8)}$ & 45.25 & $\mathrm{~cm}$ \\
\hline
\end{tabular}


To illustrate the SMC, we present the results concerning the tracking of a circular trajectory. Figure 6 shows the tracking of a circular trajectory in the track the desired 3D plane, this last, we have found that, the end-effector follows the reference trajectory with high performance (small error; between the real and the reference paths); as see in figure 6a. Figure 7 shows the time evolution of the cable lengths. we can observe the symmetry on cable lengths for (L1,L2,L3 and L4) and concerning the (L5,L6,L7 and L8) we note that, the lengths of this cables are fixed because the height $\mathrm{Z}$ is fixed (the curve in the plane $\mathrm{x}, \mathrm{y}$ ), that is to say in this means that the tensions should always be positive and be between $T_{\min }$ and $T_{\max }$, because the sliding mode control applied always cheek the role to requires adequate electrical voltages to the motors generating tensions on the cables [20]. In addition, concerning the error, we can say that is acceptable error between (3.6-3.7*10-3m).
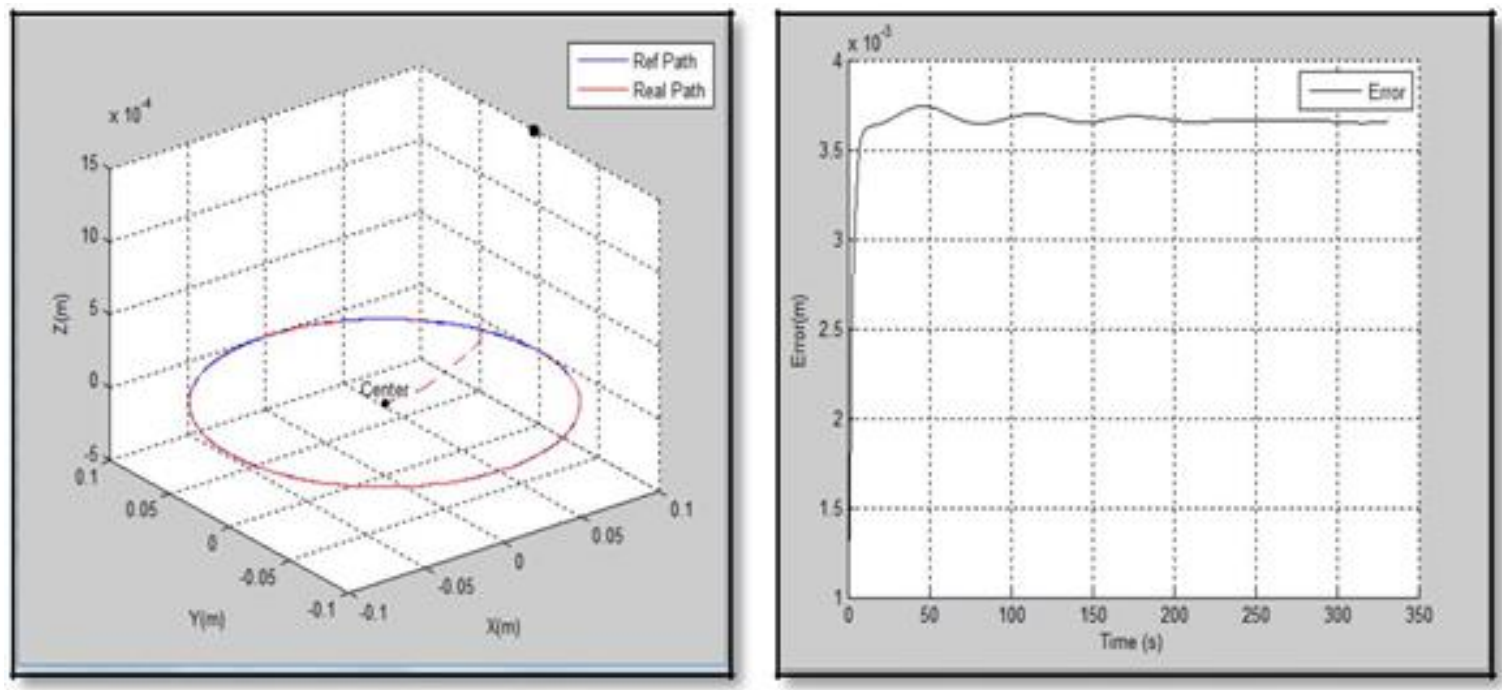

Figure 6. Tracking of circular trajectory: a) Position of end effector in 3D plane; b) position error

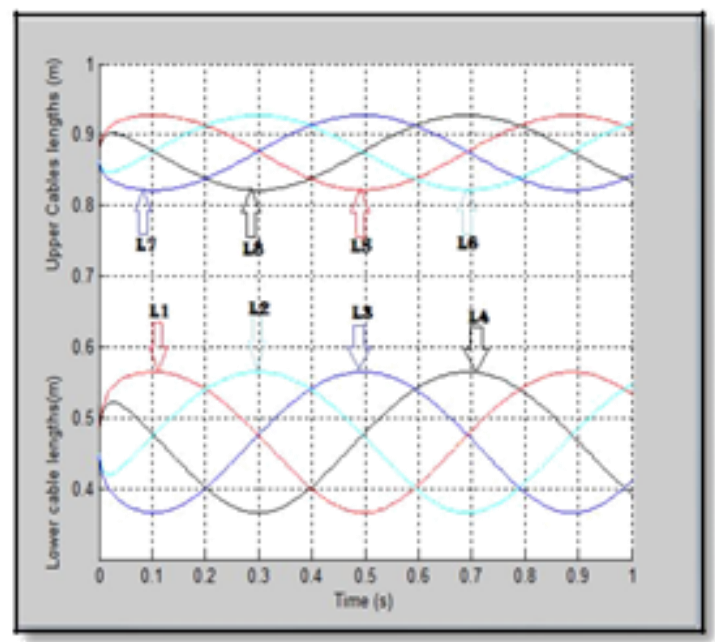

Figure7. Time evolution of cable lengths. 

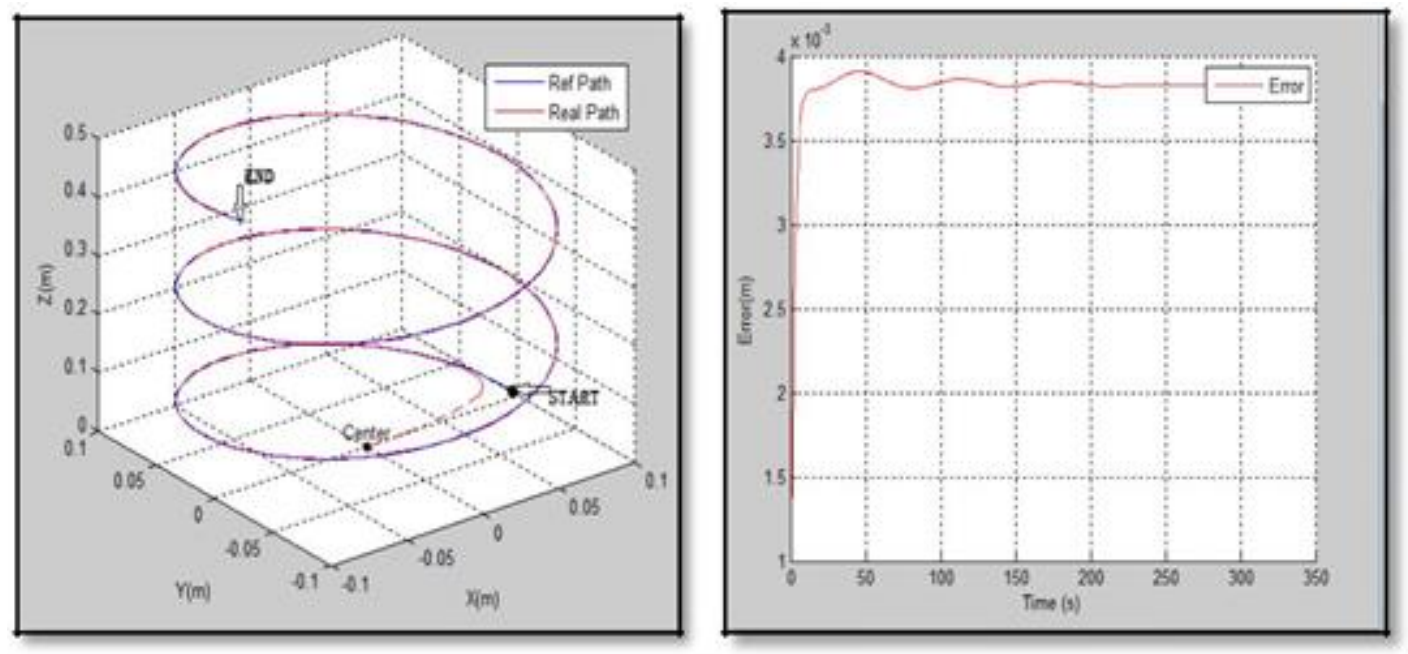

Figure 8. Tracking of a spiral trajectory:

a) Position of the end-effector in 3D plane; b) Position error.

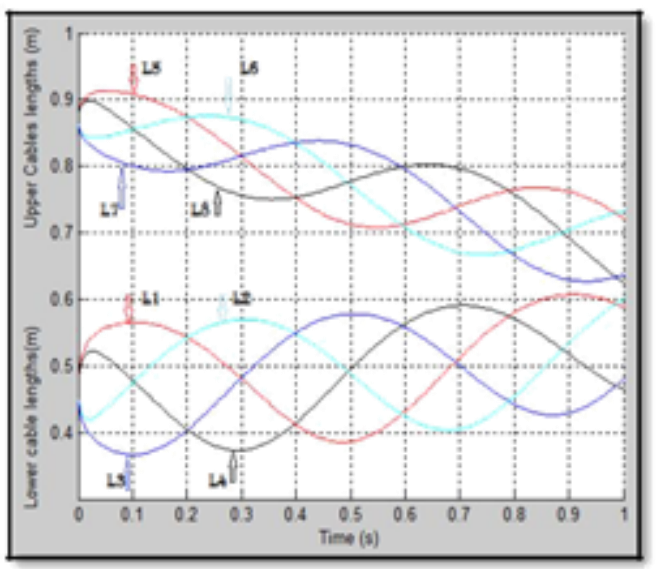

Figure 9. Time evolution of cable lengths

The analysis of the previous presented simulation results confirm the possibility to apply successfully the proposed sliding mode controller for curve shape tracking in case of relatively low and high speed. On the other hand, if we see the curve of figure $8 \mathrm{~b}$ ), we notice that, the tracking error is very low (between 3.8-3.9 mm). And comparing the two errors, figure 10, we see a very little difference of $0.1 \mathrm{~mm}$ (high controller efficiency). Concerning the cables lengths, we see that, the lower cable lengths increase relatively to the upper ones, which decrease: in order to draw a spiral path. 


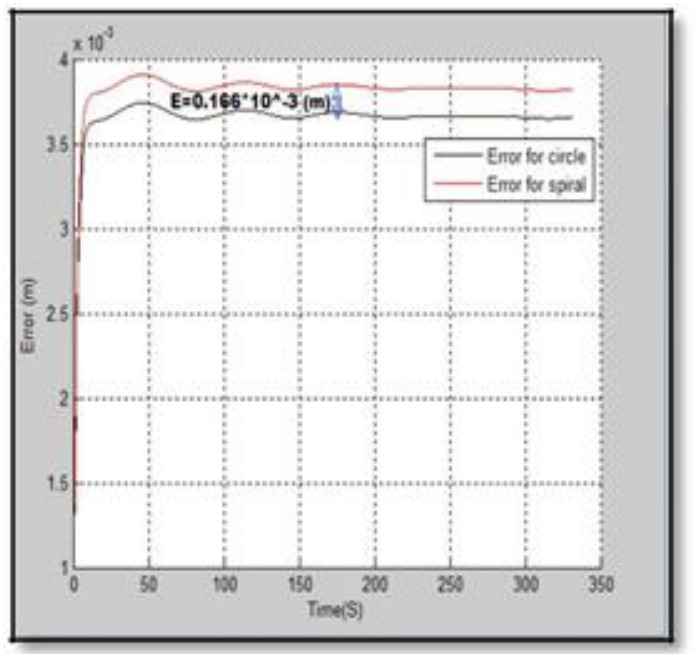

Figure 10. Comparison between the circular trajectory and the spiral trajectory errors.

To clarify more the tracking performance of our controller, we present the example of tracking one of difficult typical trajectory such as a square trajectory characterized by right angles, as in figure 11, it is clear that the sliding mode is a good tracking.

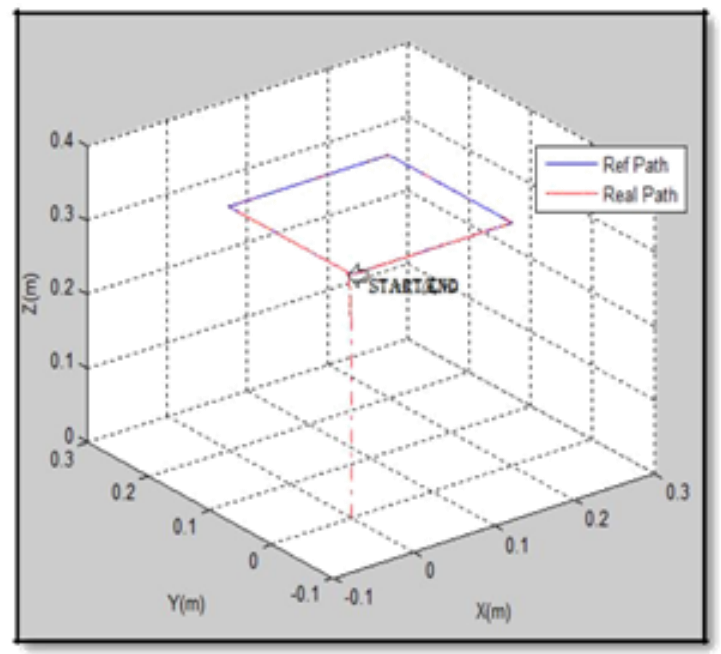

Figure 11. Plot of a square trajectory. 


\section{CONCLUSIONS}

In this paper, an adaptive controller based on sliding mode control has been successfully applied for 3D cable parallel robot. Details and algorithm are presented briefly. Specific simulations have been carried out in Matlab environment. In this point, simulation results demonstrated that the sliding mode controller has positive stretching influences on the stability of the system in spite of the chattering phenomenon. The proposed control also shows a better performance as comparing with a PID controller in most operation conditions. For further work, cable's flexibility in such robots is another source of error, which can be considered in other studies.

\section{REFERENCES}

[1] Ceccarelli M. Fundamentals of Mechanics of Robotic Manipulation. International Series on Microprocessor-Based and Intelligent Systems Engineering. 2004;27: 73240.

[2] Ceccarelli M, Romdhane L. Design issues for human-machine platform interface in cable-based parallel manipulators for physiotherapy applications. Journal of Zhejiang. 2010; 11:231-239.

[3] Alireza A, Mehdi V. Sliding Mode Control of a Cable-driven Robot via DoubleIntegrator Sliding Surface. International Conference on Control, Robotics and Cybernetics. 2012;43: 1-7.

[4] Ceccarelli M,Ottaviano E,Tavolieri C.Experimental Activity on Cable-Based Parallel Manipulators:Issues and Results at LARM in Cassino. 2nd International Congress Design and Modelling of Mechanical Systems, Monastir. 2007; 27:12-18.

[5] Surdilovic D, Zhang J,Bernhard R, String M. Wire-robot technology for safe, flexible and human-friendly gait rehabilitation. International Conference on Rehabilitation Robotics. 2007; 10:446-453.

[6] Alp AB,Agrawal SK. Cable suspended robots: design, planning and control. IEEE International conference of robotics and automation.2002; 4:4275-4280.

[7] Mirjana F. The Importance of Modelling an Aerial Robotic Camera. Scientific Technical Review. 2012;62:28-37.

[8] Gosselin C, Poulin R, Laurendeau D. A Planar Parallel 3-DOF cable-driven haptic interface. 12th World Multi-Conference on Systemics, Cybernetics and Informatics. 2008;12:266-271.

[9] Williams RL. Novel cable-suspended RoboCrane support, International Journal of Industrial Robot. 2005;32: 326-333.

[10] Ceccarelli M. Problems and Experiences on Cable- Based Service Robots for Physiotherapy Applications. New Trends in Medical and Service Robots. 2013; 16:27- 42 .

[11] Hernández MEE, Ceccarelli M, Carbone G, López CCS, Jáuregui CJC. A Characterization of Milli-CaTraSys System with a Parallel Manipulator Architecture. International Journal Mechanics Based Design of Structures and Machines.2010;38: 25-49. 
[12] Zi B, Duan BY, Du JL, Bao H. Dynamic modeling and active control of a cablesuspended parallel robot. Mechatronics. 2008;18: 1-12.

[13] Carbone, G, Aróstegui C, Ceccarelli M, Altuzarra O. A study of feasibility for a limb exercising device. Mechanisms and Machine Science. 2017;47: 11-21.

[14] Gallina P, Williams RL. Planar Cable-Direct-Driven Robots Part I: Kinimatics and Statics. Design Technical engineering conferences.2001; 27:1-9.

[15] Williams RL,Vadia J. Planar Translational Cable-Direct-Driven Robots: Hardware Implementation. Design Technical Engineering Conferences. 2003;29: 2-6.

[16] Fernini B, Temmar M, Noor MM. Toward a dynamic analysis of bipedal robots inspired by human leg muscles. Journal of Mechanical Engineering and Sciences. 2018;12:3593-3604.

[17] Fouad I,LAkhdar K. Comparison Performance between PID and PD Controllers for Cable Based Robots.World Journal of Engineering . 2014;11: 543-556.

[18] Shiler Z, Filter S, Dubowski S.Time op-timal paths and acceleration lines of robotic manipulators. International Conference of Decision and Control. 1987; 27:98-99.

[19] Young KD. A control engineer's guide to sliding mode control. IEEE Transactions on Control Systems Technology.1999;7: 328-342.

[20] Vafaei A, Khosravi MA, Taghirad HD. Modeling and control of cable driven parallel manipulators with elastic cables: Singular perturbation theory. International Conference on Intelligent Robotics and Applications. 2011; 7101: 455-464.

[21] Abdelouahab Z, Billel B. Sliding mode versus PD control for cable-based robot. World Journal of Engineering. 2014;11: 287-296. 